\title{
2. Bounded Representations on a Topological Vector Space and Weak Almost Periodicity.
}

\author{
By Kôji SHIgA \\ (Received Jan. 30, 1956)
}

\section{Introduction.}

In 1935, Bochner and v. Neumann [2 $]^{1)}$ established a theory of vector-valued almost periodic functions, i.e. almost periodic functions having their values in a topological vector space. Since then the notion of the topological vector space has been generalized and brought finally to its definitive form by Bourbaki [3]. Now, as the theory of complex-valued almost periodic functions may be considered as a theory of bounded representations of groups, it would be natural to ask for an analogous interpretation of the theory of vector-valued almost periodic functions. In this paper we shall give such an interpretation to the theory of Bochner-v. Neumann rewritten on the ground of Bourbaki's definition of the topological vector space.

This paper is divided into six sections: $\S 1$. Preliminaries, $\S 2$. Bounded representations , §3. Formulation of the main theorems, $\$ 4$. Relations between the main theorems, $\S 5$. A proof of Theorem $1, \S 6$. Consequences and supplementary remarks.

In $\S 1$ we give a precise definition of the almost periodic function on a group $G$ with the values in a locally convex vector space $L$, and in $\S 2$, that of bounded representations of $G$ on $L$ and allied definitions and propositions. In $\$ 3$ we introduce the notion of composition series (Definition 5), which seems to be of importance to our theory, in terms of which we formulate Theorem 1, one of our main theorems. We give five main theorems in close mutual relations: $1,2,3,4,4^{\prime}$, of which $4^{\prime}$ is nothing but the main result of the classical theory of complex-valued almost periodic functions. The logical relations between these theorems are indicated by a diagram at the end of $\$ 3$, and are proved in $\S 4$. At one step (numbered as 3)) we use a known result of Mackey and Arens [1], [10]. Although all our main theorems are thus reduced in a sense to the classical theorem $4^{\prime}$, it may be of interest from the viewpoint of the representation theory to give a direct proof of Theorem 1 . We shall give such a proof in $\S 5$. The final $\S 6$ is devoted to an exposition of some consequences of our main theorems and of some problems concerning our theory. In particular, the following problem remains open to the author: Does an analogue of Jordan-Hölder's theorem hold on our composition series?

The author wishes to express his hearty thanks to Professors Y. Mimura and S. Iyanaga for their valuable helps and kindly encouragements in this investigation.

1) Numbers in brackets refer to the bibliography at the end of the paper. 


\section{Preliminaries.}

We recall first some fundamental notions on topological vector spaces. Let $L$ be a topological vector space in the sense of Bourbaki [3] over the complex number field $C$, which we shall assume throughout this paper to satisfy the $T_{2}$-separation axiom. We use freely such elementary properties of $L$ as given in [3]. A subset $B$ of $L$ is called bounded, if, for any neighborhood $U$ of $0, B$ is contained in $\lambda U$, where $\lambda$ is a suitable positive number. Regarding topologies of the dual space $L^{*}$ of $L$, we are mainly interested in the strong topology: the topology of uniform convergence on all bounded subsets of $L$. Also we have occasion to consider the weak topology of $L^{*}$ : the topology of pointwise convergence on $L$. When we shall refer to $L^{*}$ simply as the dual space of $L, L^{*}$ is to be considered as a topological vector space with the strong topology. In case where $L$ is a locally convex vector space, the dual space $L^{* *}$ of $L^{*}$ contains the subspace $\dot{L}$, which is algebraically, but not always topologically, isomorphic to $L$. If $L^{* *}=\dot{L}, L$ is called semi-reflexive ; furthermore, if $L^{* *}$ is isomorphic to $L$ as topological vector spaces, $L$ is called reflexive. For any subset $S$ of $L, S^{+}$denotes the weakly-closed subspace of $L^{*}$, defined by $S^{\perp}=\{f ; f(x)=0 \text { for } x \in S\}^{2)}$. Correspondingly, for any subset $\tilde{S}$ of $L^{*}, \tilde{S}^{\perp}$ denotes the closed subspace of $L$, defined by $\tilde{S}^{-\perp}=\{x ; f(x)=0$ for $f \in \widetilde{S}\}$.

For later use, we consider locally convex vector spaces with the property that the convex-hull of any compact subset has the compact closure; we cite this property as the property (C). It is obvious that any quasi-complete space ${ }^{3}$ has the property $(C)$. As a consequence of Mackey-Arens's theorem, the following fact is known [4]: Let $L$ have the property $(C)$, and let $L^{* C}$ be the dual space of $L$ endowed with the topology of uniform convergence in all compact subsets on $L$ (Mackey's topology). Then the dual space of $L^{* C}$ is $L$, and $L^{* C}$ is semi-reflexive. A little more strengthened property $(T)$ than the above property $(C)$ will be also used: A locally convex vector space is said to have the property $(T)$, if the closure of the convex-hull of any totally bounded subset is compact.

A usual definition of complex-valued almost periodic function on a group $G$ is in an obvious manner extended to the case where the values of the functions lie in a locally convex vector space [2].

Specifically: Let $L$ be a locally convex vector space. Let $L_{b}^{G}$ be the set consisting of all functions $f$ 's from $G$ into $L$ such that $f(G)$ is a bounded subset in $L$. A topology of $L_{b}^{G}$ can be defined as follows: for every neighborhood $U$ of 0 , put $\tilde{U}$ for the set of all $x \in L_{b}^{c t}$ with a range $\subset U$; we adopt the set of all $\tilde{U}$ as a base of neighborhoods at 0 of $L_{b}^{G}$. Then, as is easily seen, $L_{b}^{G}$ becomes a locally convex vector space, and if $L$ is complete or quasi-complete, then $L_{0}^{G}$ has the same property. A function $x(a)$ from $G$ into $L$ is called almost periodic if the set $x_{b}(a), b \in G$, forms

2) We denote by $\{x ; P(x)\}$ the set consisting of all elements $x$ 's with the property $P(x)$.

3) A locally convex vector space is called quasi-complete, if any bounded closed subset is complete. 
a totally bounded subset in $L_{b}^{G}$, where $x_{b}(a)=x(a b)$. It is obvious that the set $\mathfrak{A}$ of all almost periodic functions forms a vector space; moreover, for any complex-valued almost periodic function $\alpha(a)$ and for any $x(a) \in \mathfrak{A}$, we have $\alpha(a) x(a) \in \mathfrak{A}$. $\mathfrak{A}$ becomes a locally convex vector space, when endowed with the relative topology induced from $L_{b}^{G}$. $\mathfrak{A}$ is closed in $L_{b}^{G}$, and hence $\mathfrak{A}$ is complete whenever $L$ is complete.

\section{Bounded Representations.}

We consider a representation of a fixed abstract group $G$. A representation $\{L, T(a)\}$ of $G$ on a linear space $L$ is, by definition, a homomorphism from $G$ into linear operators on $L$ such that $a \longrightarrow T(a)$. Given a representation $\{L, T(a)\}$, any invariant subspace $L_{1}$ clearly gives rise to a representation which is obtained by restricting $T(a)$ to $L_{1}$; we denote this representation by $\left\{L_{1}, T(a)\right\}$. Similarly, we denote the quotient representation by $\left\{L / L_{1}, T(a)\right\}$. We are mainly interested in representations on a topological vector space; in this case the operators $T(a)$ are assumed to be continuous.

Definition 1. Let $\{L, T(a)\}$ be a representation of $G$ on a locally convex vector space $L . \quad\{L, T(a)\}$ is called bounded, if there exists a base $\mathfrak{U}$ of neighborhoods at 0 consisting of convex sets $U_{\alpha}$ 's $(\alpha \in \Gamma)$ with the following property:

For any $\alpha$ we can find a $\rho_{\alpha}$ such that

$$
T(a) U_{\alpha} \subset \rho_{\alpha} U \text { for every } a \in G \text {. }
$$

$\{L, T(a)\}$ is called uniformly bounded, if, in addition, $\rho_{\alpha}$ is so chosen as to be independent of $\alpha$.

It is obvious that in this definition every $U_{\alpha}$ can be always supposed to be closed and cerclé : $e^{i \theta} U \subset U$ for any real $\theta$. If $\{L, T(a)\}$ is a bounded representation, then, for any invariant subspace $L_{1},\left\{L_{1}, T(a)\right\}$ and $\left\{L / \bar{L}_{1}, T(a)\right\}$ are also bounded representation, where $\bar{L}_{1}$ means the closure of $L_{1}$. In a particular case where $L$ is a normed space, it is easily verified that $\{L, T(a)\}$ is bounded if and only if we have $\sup _{a \in G}|T(a)| \leqq \mu$ for some $\mu>0$; hence in this case the notion of boundedness is not distinct from the one of uniform boundedness.

Let $\mathfrak{H}=\left\{U_{\alpha}\right\}_{\alpha} \in \Gamma$ have the same meaning as stated in Definition 1 . As is well known, if we put $p_{\alpha}(x)=\inf _{\rho>0, x \in \rho U_{\alpha}} \rho, p_{\alpha}(x)$ defines a semi-norm on $L$, and the set $P$ of $p_{\alpha}$ 's $(\alpha \in \Gamma)$ induces in a natural manner the proper topology on $L$. In what follows, whenever we deal with a bounded representation, the notations $\mathfrak{H}=\left\{U_{\alpha}\right\}$ and $P=\left\{p_{a}\right\}$ are to be used in this sense. Also, the notation $\rho_{0}$ is constantly used for the uniformly bounded representations, which denotes a positive number taken common to all $\rho_{\alpha}$ in the definition: $T(a) U_{\alpha} \subset \rho_{0} U_{\alpha}$.

Proposition 1. Let $\{L, T(a)\}$ be a bounded representation. If we put $P_{\alpha}=p_{\alpha}^{-1}(0)$, then $P_{\alpha}$ is an invariant closed subspace of $L$.

Proof. Clearly $P_{\alpha}$ is a closed subspace. Since $x \in P_{\alpha}$ is equivalent to $x \in \rho U_{\alpha}$ for every $\rho>0$, we have $T(a) P_{\alpha} \subset \rho T(a) U_{\alpha}$ for every $\rho>0$. Hence, by Definition 1 , 
$T(a) P_{\alpha} \subset \rho_{\alpha} U$, which implies $T(a) P_{\alpha} \subset P_{\alpha}$.

According to this proposition, we may consider the representation $\left\{L / P_{\alpha}, T(a)\right\}$ for each $\alpha$, which provides a bounded representation on the normed space $L / P_{\alpha}$. Let $\|T(a)\|_{\alpha}$ be the norm of $T(a)$, considered as an operator on $L / P_{\alpha}$; then we obtain $\sup _{a \in G} \mid T(a) \|_{\alpha} \leqq \rho_{\alpha}$, if $T(a) U_{\alpha} \subset \rho_{\alpha} U_{\alpha}$ for every $a \in G$. Therefore, the uniform boundedness of $\{L, T(a)\}$ means that each bounded representation $\left\{L / P_{\alpha}, T(a)\right\}$ has a common bound $\rho_{0}$ to all $\alpha$ : $\sup _{a \in G} T(\alpha) \|_{\alpha} \leqq \rho_{0}$.

Proposition 2. Let $\{L, T(a)\}$ be a bounded representation. Then, for any bounded subset $B$ of $L \underset{a \in G}{\bigcup} T(a) B$ is also a bounded subset of $L$.

Proof. Take any neighborhood $U$ of 0 , and select an $\alpha_{0}$ such that $U_{\alpha_{0}} \subset U$, $U_{\alpha_{0}} \in \mathbb{l l}$. For a suitable number $\mu>0$, we have $\mu B \subset U_{\alpha_{0}}$, so that $\left(\mu / \rho_{\alpha_{0}}\right) T(a) B$ $\subset U_{\alpha_{0}} \subset U$ for every $a \in G$, which proves our assertion.

Assume that a representation $\{L, T(a)\}$ is given. According to a usual manner, we define the adjoint operator $T^{*}(a)$ of $T(a)$ by $T^{*}(a) f(x)=f(T(a) x)$. It is evident that $T^{*}(a)$ is a continuous operator and that $\left\{L^{*}, T^{*}(a)\right\}$ gives an anti-representation of $G$.

Proposition 3. Let $\{L, T(a)\}$ be a bounded representation. Then the anti-representation $\left\{L^{*}, T^{*}(a)\right\}$ is uniformly bounded.

Proof. Put $U^{*}(C, \varepsilon)$ for the subset of $L^{*}$, defined by $U^{*}{ }_{(C, \approx)}=\left\{f ; \sup _{x \in C}: f(x) \mid<\varepsilon\right\}$ where $C$ is a bounded subset of $L$. Observe that the family of all the subsets of $L^{*}$ with a form $U^{*}(C, \varepsilon)$ gives rise to a base of neighborhoods at 0 of $L^{*}$. Suppose that a $U^{*}(C, \varepsilon)$ be given. By Proposition 2, $\underset{a \in G}{\bigcup} T(a) C=C_{1}$ is a bounded subset of $L$, so that $U^{*}\left(C_{1}, \varepsilon\right)$ is also a neighborhood of 0 . It is obvious that

$$
U^{*}\left(C_{1, \varepsilon)} \subset U^{*}(C, \varepsilon) \text { and } T^{*}(a) U^{*}{ }_{\left(C_{1}, s\right)}=U^{*}\left(C_{1, \varepsilon}\right)\right. \text {. }
$$

It follows that convex neighborhoods with a form $U^{*}(u T(a) C, s)$ give a base of neighborhoods of 0 , and that for each such a neighborhood $U^{*}$ we have

$$
T^{*}(a) U^{*}=U^{*} \quad \text { for } \quad a \in G \text {. }
$$

This means that $\left\{L^{*}, T^{*}(a)\right\}$ is a uniformly bounded representation.

Accordingly, if $\{L, T(a)\}$ is bounded, then $\left\{L^{*}, T^{*}(a)\right\},\left\{L^{* *}, T^{* *}(a)\right\}, \cdots \cdots$, etc. yield uniformly bounded representations. The anti-representation $\left\{L^{*}, T^{*}(a)\right\}$ is called the conjugate representation of $\{L, T(a)\}$.

Definition 2. A bounded representation $\{L, T(a)\}$ is called weakly almost periodic (abbreviated: w.a.p.) if $f(T(a) x)$ is an almost periodic function on $G$ for any $x \in L$ and $f \in L^{*}$.

Definition 3. A uniformly bounded representation $\{L, T(a)\}$ is called totally bounded, if $\bigcup_{a \in G} T(a) x$ is a totally bounded subset of $L$ for each $x \in L$.

It is a well-known fact that in finite-dimensional cases any bounded representation becomes w.a.p. and totally bounded.

Proposition 4. A totally bounded representation $\{L, T(a)\}$ is w.a.p.

Proof. We shall prove that $f_{0}\left(T(a) x_{0}\right)$ is almost periodic. Set $U_{s}=\left\{x ;\left|f_{0}(x)\right|\right.$ 
$<\varepsilon\}$, and take a $U_{\alpha}$ from $\mathfrak{u}$ such that $\rho_{0} U_{\alpha} \subset U_{c}$, where $\rho_{0}$ is such a positive number as was remarked previously. $M_{x_{0}}=\left\{T(a) x_{0} ; a \in G\right\}$ being totally bounded, there exist $a_{1}, \cdots \cdots, a_{n}$ such that, given any element $a$, we have

$$
T(a) x_{0}-T\left(a_{i}\right) x_{0} \in U_{\alpha}
$$

for at least one $a_{i}$. Hence

$$
T(b a) x_{0}-T\left(b a_{i}\right) x_{0} \in \rho U_{a} \subset U_{\epsilon}
$$

for every $b \in G$, so that

$$
f_{0}\left(T(b a) x_{0}\right)-f_{0}\left(T\left(b a_{i}\right) x_{0}\right)<\varepsilon ;
$$

this means that $f_{0}\left(T(a) x_{0}\right)$ is right almost periodic. Since the almost periodicity of functions is equivalent to the right one, it follows that $f_{0}\left(T(a) x_{0}\right)$ is almost periodic.

One of the most important example of totally bounded representations is the regular representation in the space of almost periodic functions on $G$. Let $\mathfrak{A}$ be a subspace in the space of almost periodic functions on $G$ taking values in $L$. An invariant closed family $\mathfrak{A}$ fulfills, by definition, the following conditions: if $x(a) \in \mathfrak{A}$, then $x_{b}(a)=x(a b) \in \mathfrak{A}$; if $x_{\alpha} \in \mathfrak{A}$ and $x_{\alpha}(a)$ converges uniformly to $x_{0}(a)$, then $x_{0} \in \mathfrak{A}$. The invariant closed family $\mathfrak{A}$ becomes a locally convex vector space by the relative topology. We define a representation of $G$ on $\mathfrak{A}$ by $R(a) x(b)=x_{a}(b)$. From the definition of the topology of $\mathfrak{A}$ it follows immediately that $\{\mathfrak{A}, R(a)\}$ is a totally bounded representation. In what follows the notation $\{\mathfrak{H}, R(a)\}$ means a representation constructed in this manner.

Definition 4. Let $\{L, T(a)\}$ be a representation of $G$ on a topological vector space. Denote by $\left\{L_{\lambda}, T(a)\right\}_{\lambda \in A}$ the family of all the finite-dimensional irreducible representations of $G$, each of whose representation space $L_{2}$ is contained in $L$. $\{L, T(a)\}$ is called completely.decomposable if $L$ is spanned by all $L_{\lambda}$ 's, $\lambda \in A$.

We observe that if a completely decomposable representation $\{L, T(a)\}$ is bounded, then the matrix coefficients of the representation which are induced from any finitedimensional irreducible subspace $L_{2}$ of $L$ are almost periodic functions, because $\left\{L_{\lambda}, T(a)\right\}$ is a bounded finite-dimensional representation.

The following lemma is almost obvious.

Lemma 1. Assume that a uniformly bounded representation $\{L, T(a)\}$ is completely decomposable; take any element $x \in L$ and any neighborhood $U$ of 0 . Then there exists a bounded finite-dimensional representation $D=\left(d_{i j}(a)\right)$, such that for any $a$

$$
T(a) x-\sum d_{i j}(a) x_{i j} \in U,
$$

where $x_{i j}$ are suitable elements, independent of $a$.

Proposition 5. Assume that a uniformly bounded representation $\{L, T(\alpha)\}$ is completely decomposable. Then $\{L, T(a)\}$ is w.a.p.

Proof. Let $x_{0}$ be any element of $L$. Take any $f_{0} \in L^{*}$ and put $U_{\varepsilon}=\left\{x ;\left|f_{0}(x)\right|\right.$ $<\varepsilon\}$. Apply the above lemma to $x_{0}$ and $U_{\varepsilon}$; then we have

and thus

$$
\begin{gathered}
T(a) x_{0}-\sum d_{i j}(a) x_{i, i} \in U_{\varepsilon}, \\
f_{0}\left(T(a) x_{0}\right)-\sum d_{i j}(a) f_{0}\left(x_{i j}\right) \mid<\varepsilon .
\end{gathered}
$$


Hence, being the uniform limit of almost periodic functions, $f_{0}\left(T(a) x_{0}\right)$ is almost periodic. This completes the proof.

\section{Formulation of the Main Theorems.}

Given a representation $\{L, T(a)\}, L$ is considered as a module over $C$ with the set of automorphisms $T(a)$ 's. From this point of view, the notion of composition series is introduced in a usual way:

Definition 5. A well-ordered family of invariant subspaces $L_{\alpha}$ 's of $\{L, T(a)\}$ is called an algebraic composition series when it satifies the following three conditions:

i) $L=L_{0} \supsetneq L_{1} \supsetneqq L_{2} \supsetneq \cdots \cdots \cdots L_{\alpha} \varsubsetneqq \cdots \cdots \cdots L_{\mathrm{c}}$.

ii) $\left\{L_{\alpha} / L_{\alpha+1}, T(a)\right\}$ is a bounded finite-dimensional irreducible representation, where the quotient linear space $L_{\alpha} / L_{\alpha+1}$ is regarded simply as a linear space; $L_{\alpha}=\bigcap_{\beta<\alpha} L_{\beta}$, if $\alpha$ is a limit number.

iii) $L_{\mathrm{s}}=\{0\}$.

In the case where $L$ is a topological vector space, if each $L_{\alpha}$ can be further assumed to be closed, then $\left\{L_{\alpha}\right\}$ is called a topological composition series; in this case, $L_{\alpha} / L_{\alpha+1}$ given in ii) may be regarded as a topological vector space with the properly induced topology.

Now we shall state the main theorems, whose proofs and consequences are discussed in the following sections.

Theorem 1. Let $\{L, T(a)\}$ be a bounded representation of $G$. Assume that we can find a subset $\tilde{S}$ of $L^{*}$ with the following properties:

i) Given $x_{0} \neq 0$, there is at least one $f \in \tilde{S}$ such that $f$ does not become identically zero on the subspace $\left[T(a) x_{0} \mid a \in G\right]^{4)}$.

ii) For any $x \in L$ and $f \in \widetilde{S}, f(T(a) x)$ is an almost periodic function on $G$.

Then $\{L, T(a)\}$ has sufficiently many topological composition series in the follow. ing sense: For any given $x_{0} \neq 0$, there is a topological composition series $L=L_{0} \supseteqq L_{1} \supsetneq$ ..... such that $x_{0} \in L_{1}$.

Theorem 2. Let $\{L, T(a)\}$ be a bounded representation satisfying the same conditions as in Theorem 1. Then there exists a total invariant subspace $\widetilde{C}^{(5)}$ of $\left\{L^{*}\right.$, $\left.T^{*}(a)\right\}$ such that $\left\{\tilde{C}, T^{*}(a)\right\}$ is completely decomposable.

Theorem 3. Let $\{L, T(a)\}$ be a totally bounded representation whose representation space $L$ has the property $(T)$. Then $\{L, T(a)\}$ is completely decomposable.

Theorem 4. Assume that the range $L$ of almost periodic functions has the property $(T)$. Then $\{\mathfrak{A}, R(a)\}$ is completely decomposable.

Theorem $4^{\prime}$. Let $\mathfrak{A}_{0}$ be an invariant closed family of complex-valued almost periodic functions. Then $\left\{\mathfrak{H}_{0}, R(a)\right\}$ is completely decomposable.

4) For a given topological vector space $L$, the notation $[x \mid P(x)]$ means the closed subspace of $L$, generated by all elements $x$ 's with the property $P(x)$.

5) A subset $\tilde{L}$ of $L^{*}$ is total if $\tilde{L}^{\perp}=\{0\}$. 
These theorems have essentially equivalent contents. In the next section we shall study their mutual relationship according to the following scheme:

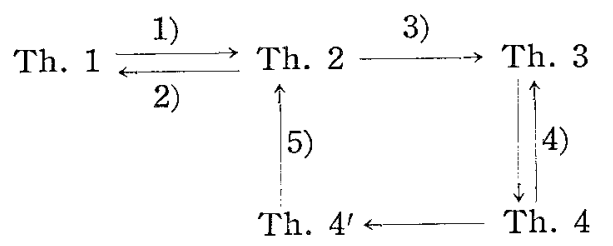

We remark that the following two implications are trivial:

Th. $3 \longrightarrow$ Th. 4 , Th. $4 \longrightarrow$ Th. $4^{\prime}$.

\section{Relations between the Main Theorems.}

1) Theorem $1 \longrightarrow$ Theorem 2 .

Assume that Theorem 1 holds. Consider the family $\Sigma$ consisting of all invariant closed subspaces $L_{\nu}$ 's of $\{L, T(a)\}$ such that each representation $\left\{L / L_{\nu}, T(a)\right\}$ is a bounded finite-dimensional representation. Observe that $\Sigma$ contains those invariant closed subspace which appears as the first member of any topological composition series. Put $\tilde{C}$ for the subspace of $L^{*}$, which is generated by all $L_{\nu}$ 's, $L_{\nu} \in \Sigma$. Since $\left\{L / L_{\nu}, T(a)\right\} \cong\left\{L_{\nu}^{\perp}, T^{*}\left(a^{-1}\right)\right\}, \check{C}$ is completely decomposable. Hence it suffices to show that $\tilde{C}$ is total, i.e. $\tilde{C}^{\perp}=\{0\}$; or equivalently, given $x_{0} \neq 0$, there are an $L_{\nu} \in \Sigma$ and an element $f \in L_{\nu}^{\perp}$ such that $f\left(x_{0}\right) \neq 0$. But the existence of such an element $f$ is an immediate consequence of Theorem 1 and the theorem of Hahn-Banach, because by Theorem 1 we can find an $L_{\nu} \in \Sigma$ such that $L_{\nu} \supsetneqq x_{0}$.

2) Theorem $2 \longrightarrow$ Theorem 1 .

We first state:

Proposition 6. Let $\{L, T(a)\}$ be a bounded representation satisfying the same conditions as in Theorem 1 . Then the conjugate representation $\left\{L^{*}, T^{*}(a)\right\}$ has a well-ordered family of invariant weakly-closed subspaces $L_{\alpha}$ 's such that

i) $0 \subsetneq \tilde{L}_{1} \subsetneq \tilde{L}_{2} \subsetneq \cdots \cdots \subsetneq \tilde{L}_{\alpha} \subsetneq \cdots \cdots . \subsetneq \tilde{L}_{\varepsilon}=L^{*}$.

ii) $\left\{\tilde{L}_{\alpha+1} / \tilde{L}_{\alpha}, T^{*}(a)\right\}$ is a bounded finite-dimensional irreducible representation.

iii) If $\alpha$ is a limit number, $\cup_{\beta<\alpha} \tilde{L}_{\beta}$ is a weakly dense subspace in $L^{*}$.

Assuming that Theorem 2 is valid, we can easily establish this proposition by piling up the finite-dimensional invariant subspaces of $\left\{L^{*}, T^{*}(a)\right\}^{6)}$. From this proof it follows that the choice of $\widetilde{L}_{1}$ is arbitrary to such a degree that the totality of $\tilde{L}_{1}$ forms a total subspace. It is easily checked that Proposition 6, combined with the preceding remark, is exactly the dual form of Theorem 1 . Hence Theorem $2 \longrightarrow$ Theorem 1 is proved.

3) Theorem $2 \longrightarrow$ Theorem 3 .

Assume that Theorem 2 holds. Let $\{L, T(a)\}$ be a w.a.p. representation whose

6) In order to complete this procedure, we require the following result: For any weaklyclosed subspace $\tilde{L}$ of $L^{*}$, we have $(\tilde{L})^{++}=\widetilde{L}$. Cf. [7], p. 23 . 
representation space $L$ satisfies the property $(T)$. Observe that $L$ is the dual space of $L^{* C}$ (Mackey's topology); putting $\tilde{S}=L$, we see that the representation $\left\{L^{* c}, T^{*}(a)\right\}$ fulfills the conditions i) and ii) stated in Theorem 1. Hence if $\left\{L^{* c}, T^{*}(a)\right\}$ is a bounded representation, we can apply Theorem 2 to $\left\{L^{* c}, T^{*}(a)\right\}$ which shows that the conjugate representation $\{L, T(a)\}$ is completely decomposable.

Accordingly, the proof of Theorem 3 is reduced to the proof of the following proposition.

Proposition 7. Let $L$ be a locally convex vector space with the property $(T)$. Let $\{L, T(a)\}$ be a totally bounded representation. Then $\left\{L^{* C}, T^{*}(a)\right\}$ is uniformly bounded.

Proof. We first prove that, for any compact subset $C$ of $L, \bigcup_{a \in G} T(a) C$ is totally bounded. Let $U$ be any given neighborhood of the origin of $L$. We take a neighborhood $V$ of the origin such that $V+V \subset\left(1 / \rho_{0}\right) U$, where $\rho_{0}$ is a positive number associated with the uniformly bounded representation $\{L, T(a)\}$. Since $C$ is compact, there exists a finite number of elements $x_{1}, \cdots \cdots, x_{n}$ in $L$ such that

$$
C \subset U_{i}\left(x_{i}+V\right)
$$

Hence

$$
\bigcup_{a} T(a) C \subset \bigcup_{i}\left(\bigcup_{a} T(a) x_{i}+\bigcup_{a} T(a) V\right)
$$

Since $\bigcup_{a} T(a) x_{i}$ is totally bunded and $\bigcup_{a} T(a) V \subset \rho_{0} V$, it follows immediately that there are elements $y_{1}, \cdots \cdots, y_{n}$ such that

$$
U T(a) C \subset U\left(y_{j}+U\right) \text {. }
$$

This means that $\cup T(a) C$ is a totally bounded subset.

Now we proceed to the proof of Proposition 7. Observe that a base of neighborhoods at 0 of $L^{* C}$ is given by the sets $\widetilde{U}_{(C, \varepsilon)}$ 's defined by $\widetilde{U}_{(C, \varepsilon)}=\left\{f ; \sup _{x \in C}|f(x)|<\varepsilon\right\}$,

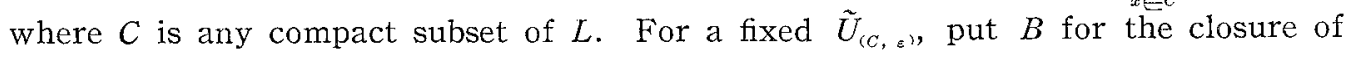
$\cup T(a) C$. Then by the above remark and the property $(T)$, we know that $B$ is a compact subset containing $C$; besides, $B$ is invariant under $T(a)$ 's. It follows that $\tilde{U}_{(B, s)}$ is a neighborhood of the origin of $L^{*}$ and that

$$
\tilde{U}_{(C, \varepsilon)} \supset \tilde{U}_{(B, \varepsilon)}, \quad T^{*}(a) \tilde{U}_{(B, \varepsilon)}=\tilde{U}_{(B, \varepsilon)}
$$

This implies that $\left\{L^{* c}, T^{*}(a)\right\}$ is uniformly bounded, which completes the proof.

4) Theorem $4 \rightarrow$ Theorem 3 .

Assume that a totally bounded representation $\{L, T(a)\}$ is given. Put $\tilde{x}(a)=T(a) x$; then the relation (1) given in the proof of Proposition 4 means that $\tilde{x}(a)$ is an almost periodic function from $G$ into $L$. Let $\mathfrak{A}_{T}$ be the subspace of almost periodic functions composed of all $\tilde{x}(a), x \in L$. Obviously $\mathfrak{A}_{T}$ is invariant under $R(a)$. Let $\Psi$ denote the correspondence of $L$ onto $\mathfrak{A}_{T}$ such that $\Psi(x)=\tilde{x}$. Then $\Psi$ is clearly linear, and moreover, for any neighborhood $U$ of 0 we have

$$
\mathfrak{A}_{T} \cap \tilde{U} \subset \Psi(U) \subset \mathfrak{H}_{T} \cap \rho_{0} \tilde{U}
$$

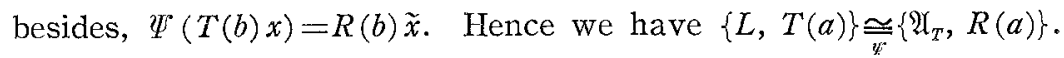

Consequently the study of totally bounded representation is essentially equivalent to the one of almost periodic function. It is easily verified that $\mathfrak{A}_{T}$ becomes a closed 
invariant family. Hence if $L$ satisfies the property $(T)$, the complete decomposability of $\{L, T(a)\}$ is a consequence of the one of $\left\{\mathfrak{U}_{T}, R(a)\right\}$.

5) Theorem $4^{\prime} \longrightarrow$ Theorem $2^{\prime}$.

Lemma 2. Let $\{L, T(a)\}$ be a bounded representation. Then, for any element $f \in L^{*}$, there is at least one $\alpha \in \Gamma$ such that $f(x)$ is identically zero for $x \in P^{(\alpha)}$, that is, $f\left(P^{(\alpha)}\right)=0^{7)}$.

Proof. Put $x_{0}$ for any element of $L$ such that $f\left(x_{0}\right) \neq 0$. Consider a neighborhood $U$ of 0 , defined by $U=\{x ; f(x)<1\}$. Then there is a $U_{\alpha_{0}} \in \mathfrak{l l}$ such that $U_{\alpha} \subset U$. Since $x_{0} / f\left(x_{0}\right)$ is not contained in $U$, we have

$$
p_{\alpha_{0}}\left(\frac{x_{0}}{f\left(x_{0}\right)}\right)=\frac{1}{f\left(x_{0}\right)} p_{\alpha_{0}}\left(x_{0}\right) \geqq 1 .
$$

This implies $x_{0} \in P^{\left(\alpha_{0}\right)}$, so that $P^{\left(\alpha_{0}\right)}$ has the required preperty.

Now we prove Theorem $4^{\prime} \longrightarrow$ Theorem 2 . Let $\{L, T(a)\}$ be a bounded representation which satisfies the condition i) and ii) in Theorem 1 . We denote by $D$ all inequivalent classes of bounded finite-dimensional irreducible representation of $G$; as a representative element of each class of $\mathscr{D}$ we can take a unitary representation. Take any representation $D^{(\alpha)}(a)=\left(d_{i j}^{(\alpha)}(a)\right)$ from $\mathscr{D}$ and put

$$
\tilde{f}_{i j}^{(\alpha)}(x)=M_{a}\left\{\overline{d_{i j}^{(a)}(a)} f(T(a) x)\right\}^{8)}
$$

where $f$ is any fixed element of $\tilde{S}$. We first show that $\tilde{f}_{i j}^{(\alpha)}$ induces an element of $L^{*}$. Since the linearily is obvious, it suffices to show that $\tilde{f}_{i j}^{(\alpha)}\left(x_{\lambda}\right) \longrightarrow \tilde{f}_{i j}^{(\alpha)}\left(x_{0}\right)$ when $x_{\lambda} \longrightarrow x_{0}$. For the sake of simplicity, put $\tilde{f}=\tilde{f}_{i j}^{(\alpha)}$. By Lemma 2, we have $\tilde{f}\left(P^{(\alpha)}\right)=0$ for an $\alpha \in \Gamma$. Hence $\tilde{f}$ may be considered as an element of $\left(L / P^{(\alpha)}\right)^{*}$. Observe the bounded representation $\left\{L / P^{(\alpha)}, T(a)\right\} ; L / P^{(\alpha)}$ being a normed space, we have $\sup _{\cos }|T(a)|_{\alpha} \leqq \mu$ for a positive number $\mu$, where $\left\{T(a) \|_{\alpha}\right.$ means the norm of $T(a)$ as an operator on $L / P^{(\alpha)}$. Put $\|\left. x\right|_{\alpha}=p_{\alpha}(x)$ and $\|\left.\tilde{f}\right|_{\alpha}=\sup _{\|\|_{\alpha} \leqq 1}|\tilde{f}(x)|$. Then it is evident that

$$
\sup _{a \in G} \tilde{f}(T(a) x) \mid \leqq \mu\|\tilde{f}\|_{\alpha} \cdot\|x\|_{\alpha} .
$$

On the other hand, if $x_{\lambda} \longrightarrow x_{0}$, we have $\mid x_{\lambda}-x_{0} \|_{\alpha}=p_{\alpha}\left(x_{2}-x_{0}\right) \rightarrow 0$. Hence by (2) and (3) we obtain

$$
\tilde{f}\left(x_{2}\right) \longrightarrow \tilde{f}\left(x_{0}\right)
$$

Now it is easily verified that $\left\{\tilde{f}_{i j}^{(\alpha)}\right\}_{i, j}$ span a finite-dimensional invariant subspace $M_{f}^{(\alpha)}$ in $\left\{L^{*}, T^{*}(a)\right\}$, and that $\left\{M_{f}^{(\alpha)}, T^{*}(a)\right\} \cong D^{(\alpha)}\left(a^{-1}\right)$ or $\cong 0$. Put $\tilde{M}$ for the subspace of $L^{*}$ which is generated by all $M_{f}^{(\alpha)}$ s, where $D^{(\alpha)}$ runs over all $\mathfrak{D}$ and $f$ over $\tilde{S}$. We wish to show that $\tilde{M}$ has the properties required in Theorem 2. From the above discussions we see that $\left\{\tilde{M}, T^{*}(a)\right\}$ is completely decomposable. Hence it is enough to prove that $\tilde{M}$ is total in $L^{*}$. Take any element $x \in L$ such that $f(x)=0$ for all $f \in \tilde{M}$. Then $\tilde{f}_{i j}^{(\alpha)}(x)=0$ for all $\alpha$ and $f \in \tilde{S}$, whence $f(T(a) x) \equiv 0$. According to the property i) of $\tilde{S}$ stated in Theorem 1 , this implies $x=0$, which completes the proof.

7) As to the notations $\Gamma$ and $P^{(\alpha)}$, see the paragraphs subsequent to Definition 1.

8) For any complex valued almost periodic function $\varphi(a)$, we denote the mean value of $\varphi(a)$ by $M_{a}\{\varphi(a)\}$. 
In conclusion, the fundamental theorem of complex-valued almost periodic functions suffices to establish the four theorems listed in the preceding section. The direct proof of Theorem $4^{\prime} \longrightarrow$ Theorem 4 was first accomplished by Bochner and v. Neumann [2] and shortly later by Maak [8]. Their methods consist essentially in constructing the mean-value $\tilde{M}_{a}$ of vector-valued almost periodic functions and using the fact that

$$
f\left(\tilde{M}_{a}\{T(a) x\}\right)=M_{a}\{f(T(a) x)\}
$$

\section{A Proof of Theorem 1.}

Although we have proved Theorem 1 by making use of the ciassical theory of almost periodic functions, it seems desirable to us to point out a way approaching Theorem 1 from the viewpoint of the representation theory; besides, the implications Th. $1 \rightarrow$ Th. $2 \longrightarrow$ Th. 3 show that a direct proof of Theorem 1 gives us a new method building up the general theory of representations. In this section we give a direct proof of Theorem 1 with this view; the theory of complex-valued almost periodic functions is not used for this proof beyond existence of the mean value. The fundamental idea of this proof is, however, closely connected with a usual proof of Theorem $4^{\prime}$.

We first consider a w.a.p. representation $\{H, U(a)\}$ of $G$ by unitary operators on a Hilbert space $H$. It is obvious that the complete decomposability of $\{H, U(a)\}$ is equivalent to the following: $H$ is representable as a direct sum of finite-dimensional subspaces $H_{\alpha}$ 's, each of which is invariant and irreducible under $U(a)$ 's, $a \in G: H=\sum \oplus H_{\alpha}$. We wish to prove :

$A$ w.a.p. unitary representation $\{H, U(a)\}$ is completely decomposable $e^{9}$.

It suffices to prove this proposition in the case where $\{H, U(a)\}$ has a cyclic vector $\xi ; H=[U(a) \xi \mid a \in G]$. Define a metric on $G$ by

$$
\operatorname{dist}_{\xi}(a, b)=\sup _{c, d} \mid(\xi, U(c a d) \xi)-(\xi, U(c a d) \xi) ! \cdot
$$

Then $G_{1}=G / K$ becomes a totally bounded group with respect to this metric, where $K$ is a normal subgroup of $G$ consisting of all elements $a$ 's such that $\operatorname{dist}_{\tilde{\xi}}(a, e)=0$. It is easily verified that $K$ is identical with the kernel of $a \rightarrow U(a)$. Therefore the representation $\{H, U(a)\}$ of $G$ can be considered as a weakly-continuous representation of $G_{1}$.

Let $\bar{G}_{1}$ be the compact group obtained by the completion of $G_{1}$; Let $a_{n} \rightarrow \bar{a}$, where $a_{n} \in G_{1}$ and $\bar{a} \in \bar{G}_{1}$. Then for any fixed $x \in H, \lim U\left(a_{n}\right) x$ exists. Put $U(\bar{a}) x$ $=\lim U\left(\bar{a}_{n}\right) x$. Then it is not difficult to see that $U(\bar{a})$ induces a unitary operator on $H$ and that $\{H, U(\bar{a})\}$ gives rise to a weakly continuous unitary representation of $\bar{G}_{1}$. Now according to the well-known fact ${ }^{10)}$ that any weakly-continuous unitary representation of a compact group is completely decomposable, we see that $\{H, U(\bar{a})\}$

9) Actually, this result will be nearly trivial if we assume Theorem 2 (Corollary 2 to Theorem 10).

10) See, for example, Proposition 1 in [13]. 
is completely decomposable, a fortiori $\{H, U(a)\}$ has the same property.

Now we proceed to the proof of Theorem 1 . Let $\{L, T(a)\}$ be a bounded representation as in Theorem 1. Put $L_{0}=L$. Assume that we have defined invariant closed subspaces $L_{\alpha}$ 's, corresponding to each ordinal number $\alpha$ which is smaller than $\alpha_{0}$. If $\alpha_{0}$ is a limit number, we define $L_{\alpha_{0}}=\cap L_{\alpha}$. Otherwise, if there exists an invariant closed subspace $L^{\prime}$ such that $L^{\prime} \subset L_{\alpha_{0}-1}$ and $\left\{L_{\alpha_{0}-1} / L^{\prime}, T(a)\right\}$ is a bounded finite-dimensional irreducible representation, we define $L_{\alpha_{0}}=L^{\prime}$. Starting from $L_{0}$, and performing this procedure as far as possible, we can get a well-ordered family of decreasing invariant closed subspaces $L_{\alpha}$ 's, which is maximal with respect to the properties i) and ii) described in Definition 5. Put $M=\cap L_{\alpha}$. We wish to establish $M=\{0\}$, which implies that $\{L, T(a)\}$ has at least one topological composition series. For this purpose, we shall lead a contradiction from the assumption $M \neq\{0\}$.

Let $x_{0}$ be a non-zero element of $M$. Let $f_{0}$ be an element of $S$ which is not identically zero on the subspace $\left[T(a) x_{0}, a \in G\right]$. For any $x, y \in M$, put

$$
(x, y)=M_{a}\left\{f_{0}(T(a) x) \overline{f_{0}(T(a) y)}\right\} .
$$

Then $(x, y)$ induces a quasi-inner product into $M$ and we have $(T(a) x, y)=\left(x, T(a)^{-1} y\right)$. Also, making use of the same considerations as in Lemma 2, we find that $\left(x_{\alpha}, x_{\alpha}\right)$ $\longrightarrow\left(x_{0}, x_{0}\right)$, when $x_{\alpha} \longrightarrow x_{0}$ in $L$. It follows that, if we set $N_{0}=\{x ;(x, x)=0, x \in M\}$, then $N_{0}$ is an invariant closed subspace of $L$ and the Hilbert space $H$ which is obtained from the completion of the linear space $\tilde{M}=M / N_{0}$ admits a unitary representation $\{H, U(a)\}$; in fact, $\{H, U(a)\}$ is defined as a natural extension of $\{\tilde{M}, T(a)\}$. We denote the canonical linear mappings between three spaces $M, \tilde{M}$ and $H$ in the following manner:

$$
\begin{aligned}
& M \longrightarrow M / N_{0} \longrightarrow H(\tilde{M}) \subset H \\
& x \longrightarrow \dot{x} \longrightarrow \ddot{x}
\end{aligned}
$$

where $H(\tilde{M})$ denotes the image of $\tilde{M}$ into $H$. Observe that the mappings $x \longrightarrow \dot{x}$, $x \rightarrow \ddot{x}, \dot{x} \rightarrow \ddot{x}$ are all continuous.

Since $(U(a) \ddot{x}, \ddot{y})=M_{b}\left\{f_{0}(T(b a) x) \overline{f_{0}(T(\bar{b}) y)}\right\}$ is an almost periodic function on $G$ for any $\ddot{x}, \ddot{y} \in H(M),\{H, U(a)\}$ is a w.a.p. representation and thus completely decomposable by Theorem 1 :

$$
H=\Sigma \oplus H_{\mu}=\sum \oplus P_{z:} H
$$

where $P_{\mu}$ means the projection operator whose range is $H_{\mu}$.

Put $H_{\mu}^{\prime}=H \ominus H_{\mu}$. Assume for a moment that $H(\tilde{M}) \cap H_{\mu_{0}}=\{0\}$ for some $\mu_{0}$. Then we could define a non-zero invariant closed subspace $\tilde{M}_{\mu_{0}}$ of $\{\tilde{M}, T(a)\}$ by

$$
\tilde{M}_{\mu_{0}}=\left\{\dot{x}: \ddot{x} \in H(\tilde{M}) \cap H_{\mu_{0}}{ }^{\prime}\right\} \text {. }
$$

$H(\tilde{M})$ being dense in $H$, we would have $\tilde{M} \supseteqq \tilde{M}_{\mu_{0}}$; besides, $\left\{\tilde{M} / \tilde{M}_{\mu_{0}}, T(a)\right\}$ were a bounded finite-dimensional representation because $\left\{\tilde{M} / \tilde{M}_{\mu_{0}}, T(a)\right\} \cong\left\{H_{\mu_{0}}, U(a)\right\}$. Define an invariant closed subspace $M_{\mu_{0}}$ of $\{L, T(a)\}$ by $M_{\mu_{0}}=\left\{x ; \dot{x} \in \tilde{M}_{\mu_{0}}\right\}$. Then we would have $M_{\mu_{0}} \subsetneq M$ and $\left\{M / M_{\mu_{0}}, T(a)\right\} \cong\left\{\tilde{M} / \tilde{M}_{\mu_{0}}, T(a)\right\}$. The existence of such an invariant closed subspace $M_{\mu_{0}}$ contradicts the maximality of $\left\{L_{\alpha}\right\}$ which was assumed above. 
Hence we obtain $H(\check{M}) \cap H_{\mu^{\prime}}=\{0\}$ for every $\mu$. This implies that for any $\mu$ the correspondence $\dot{x} \longrightarrow P_{\mu} \ddot{x}$ induces a linear isomorphism between $\tilde{M}$ and $H_{\mu}$, whence $\tilde{M}$ is finite-dimensional. Moreover, by this isomorphism we have $\{\tilde{M}, T(a)\} \cong\left\{H_{\mu}, U(a)\right\}$. Hence $\left\{M / N_{0}, T(a)\right\}$ is a bounded finite-dimensional irreducible representation. From the assumption, this is possible only when $M=N_{0}$. But $M=N_{0}$ means in particular that

$x_{0} \in N_{0}$, so that $\bigcup_{a \in G} T(a) x_{0} \subset N_{0}$. Therefore, for any element $y$ belonging to $\bigcup_{a \subseteq G} T(a) x_{0}$, we have $(y, y)=M_{a}\left\{\left|f_{0}(T(a) y)\right|^{2}\right\}=0$, whence $f_{0}(y)=0$. This implies that $f_{0}(x)$ is zero on $\left[T(a) x_{0} \mid a \in G\right]$, which is contradictory to the choice of $f_{0}$. From this contradiction we conclude $M=\{0\}$, as we wished to prove.

It remains to prove Theorem 1 that, given $x_{0} \neq 0$, we can find an invariant closed subspace $L_{1} \neq x_{0}$ such that $\left\{L / L_{1}, T(a)\right\}$ is a bounded finite-dimensional irreducible representation of $G$. In order to show the existence of such an $L_{1}$, we take an $f_{0} \in \widetilde{S}$ with the property

$$
M_{a}\left\{\mid f_{0}\left(T(a) x_{0}\right)^{2}\right\} \neq 0
$$

this is possible, according to the property i) of $\widetilde{S}$ stated in Theorem 1 . Making use of this $f_{0}$ and carrying out the embedding procedure of $\{L, T(a)\}$ into $\{H, U(a)\}$ as we have done above, we see that $\ddot{x}_{0} \neq 0$ and so, for at least one $\mu, P_{\mu} \ddot{x}_{0} \neq 0$, equivalently $\ddot{x}_{0} \in H_{\mu}{ }^{\prime}=H \ominus H_{\mu}$; here $H=\sum \oplus H_{\mu}=\sum \oplus P_{\mu} H$ denotes a complete decomposition of $\{H, U(a)\}$. Hence if we put $L_{1}=\left\{x ; \ddot{x} \in H_{\mu}^{\prime}\right\}, L_{1}$ has the desired property. This completes the proof of Theorem 1 .

\section{Consequences and Supplementary Remarks.}

Theorem 1, together with Proposition 5, yields the following result:

Theorem 5. A uniformly bounded and completely decomposable representation has sufficiently many topological composition series.

Proposition 8. Let $\{L, T(a)\}$ be a w.a.p. representation. Then, for any invariant closed subspace $M$, there is a topological composition series of $\{L, T(a)\}$ in which $M$ appears.

Proof. The proof is easily obtained by applying Theorem 1 to $\{L / M, T(a)\}$ and $\{M, T(a)\}$, respectively.

Proposition 9. If $\{L, T(a)\}$ has a topological composition series, then any invariant closed subspace has a topological composition series.

Proof. Let $\left\{L_{\alpha}\right\}, L_{0}=L$, be a topological composition series of $\{L, T(a)\}$. An observation of a family of invariant closed subspaces $\left\{L_{\alpha} \cap M\right\}$ immediately yields our assertion.

The following problem remains open to the author. An affirmative answer to it would give a result corresponding to Jordan-Hölder's theorem.

Is any topological composition series of $\{L, T(a)\}$ uniquely determined up to the order?

More precisely, let $L=L_{0} \supseteqq L_{1} \varsubsetneqq \cdots \cdots \cdots L_{\alpha} \varsubsetneqq \cdots \cdots \cdots\{0\}$ be a topological composition 
series; for any equivalent class $D$ of bounded finite-dimensional irreducible representation of $G$, put $((D))$ for the cardinal number, possibly including 0 , which shows the number of $\alpha$ such that $D \cong\left\{L_{\alpha} / L_{\alpha+1}, T(a)\right\}$. Then the above problem is expressed as follows: For a fixed representation $\{L, T(a)\}$, is each $((D))$ independent of the choice of a topological composition series? The author does not know even whether the family of $D$ such that $((D)) \neq 0$ is uniquely determined by $\{L, T(a)\}$.

Now we shall consider a converse of Theorem 1 :

Theorem 6. Let $\{L, T(a)\}$ be a bounded representation. In order that $\{L, T(a)\}$ has sufficiently many topological composition series it is necessary and sufficient that $L^{*}$ has a subset $\widetilde{S}$ with the properties stated in Theorem 1.

Proof. It suffices to prove the necessity of the condition. Let $x_{0}$ be any nonzero element of $L$. Consider a topological composition series $\left\{L_{\alpha}\right\}$ of $\{L, T(a)\}$ such that $L_{1} \neq x_{0}$. From the theorem of Hahn-Banach it follows that there is an $f \in L_{1}^{\perp}$ with $f\left(x_{0}\right) \neq 0 ; f$ can be considered as an element of $\left(L / L_{1}\right)^{*}$. Since $\left\{L / L_{1}, T(a)\right\}$ is a bounded finite-dimensional representation, $f(T(a) x)$ is almost periodic in $a$ for any $x \in L$. Thus we have proved that for any $x_{0} \neq 0$ we can find an element $f \in L^{*}$ with the properties i) and ii) stated in Theorem 1 .

Here we state a problem related to this theorem: If $\{L, T(a)\}$ is a bounded representation with a topological composition series, has $\{L, T(a)\}$ sufficiently many topological composition series?

By a slight modification of the proof of Theorem 1 given in $\S 5$, we obtain

Theorem 7. Let $L$ be a linear space regardless of the topology and $\{L, T(a)\}$ an arbitrary representation of $G$. Assume that the conjugate space $L^{*}$ has a subset $\tilde{S}$ such that

i) For any $x_{0} \neq 0$, there is an $f \in \tilde{S}$ with the property that $f$ does not become identically zero on $\bigcup_{a \in G} T(a) x_{0}$.

ii) $f(T(a) x)$ is an almost periodic function on $G$ for any $x \in L$ and $f \in \tilde{S}$. Then $\{L, T(a)\}$ has sufficiently many algebraic composition series.

From the existence of composition series we can deduce immediately the finite dimensionality of irreducible representations.

Theorem 8. Let L be a linear space. Let $\{L, T(a)\}$ be an algebraically-irreducible representation of $G$; that is, let $\{L, T(a)\}$ have no proper invariant subspace. Assume that there are an $x_{0} \in L$ and an $f_{0} \in L^{*}$ such that $f_{0}\left(T(a) x_{0}\right)$ is an almost periodic function on $G$ which is not identically zero. Then $L$ is finite-dimensional.

Theorem 9. Let $\{L, T(a)\}$ be a bounded topologically-irreducible representation; that is, let $\{L, T(a)\}$ have no proper invariant closed subspace. Assume that $\{L, T(a)\}$ fulfills the same conditions as in the above theorem. Then $L$ is finite-dimensional.

Proof. Theorem 8 is obtained from Theorem 7 , if we can show that $\{L, T(a)\}$ satisfies the conditions i) and ii) of Theorem 7 relative to $\widetilde{S}=\left\{f_{0}\right\}$. This is, however, obvious from the hypothesis, combined with the fact that any element of $L$ is expressed as $\sum \alpha_{i} T\left(a_{i}\right) x_{0}$. Similiarly Theorem 9 will become an immediate conse- 
quence of Theorem 1, if the conditions i) and ii) of Theorem 1 are checked relative to $\tilde{S}=\left\{f_{0}\right\}$. Since the condition i) is clear from the topological irreducibility, we show the validity of the condition ii). From Proposition 1 and the topological irreducibility of $\{L, T(a)\}$, it follows that $L$ is not only a locally convex vector space but also a normed space. Hence for any $x \in L$ there are $y_{n}$ 's with the form $\sum \alpha_{i} T\left(a_{i}\right) x_{0}$ such that $\left\|x-y_{n}\right\| \rightarrow 0$, which implies that $f_{0}(T(a) x)$ is the uniform limit of $f_{0}\left(T(a) y_{n}\right)$. On the other hand, by the hypothesis $f_{0}\left(T(a) y_{n}\right)$ is almost periodic, whence $f_{0}(T(a) x)$ is almost periodic. This completes the proof.

Concerning a w.a.p. representation, by Theorems 1 and 2 we get

Theorem 10. Let $\{L, T(a)\}$ be a w.a.p. representation. Then

i) $\{L, T(a)\}$ has sufficiently many topological composition series.

ii) $1\left\{L^{*}, T^{*}(a)\right\}$ has sufficiently many topological composition series.

ii) $2\left\{L^{*}, T^{*}(a)\right\}$ has a total invarinat subspace $\tilde{C}$ such that $\left\{\tilde{C}, T^{*}(a)\right\}$ is completely decomposable.

iii) $\left\{L^{* *}, T^{* *}(a)\right\}$ has a total invariant subspace $\tilde{\tilde{C}}$ such that $\left\{\tilde{\tilde{C}}, T^{* *}(a)\right\}$ is completely decomposable.

Corollary 1. Let $L$ be a semi-reflexive space. Then a w.a.p. representation $\{L, T(a)\}$ is complete decomposable.

Corollary 2. Let $L$ be a reflexive space, and $\{L, T(a)\}$ a bounded representation Then $\{L, T(a)\}$ and $\left\{L^{*}, T^{*}(a)\right\}$ are both complete decomposable if and only if $\{L$, $T(a)\}$ is w.a.p.

Example. Consider a fixed compact group $G$ and the regular representation of $G$ induced in $L^{1}(G)$, where $L^{1}(G)$ is the Banach space composed of integrable functions with respect to the Haar measure. We denote this representation by $\left\{L^{1}(G)\right.$, $R(a)\}: \quad R(a) x(b)=x(b a)$. Then $\left\{L^{1}(G), R(a)\right\}$ is clearly w.a.p. and complete decomposable; its conjugate representation is $\left\{L^{\infty}(G), L(a)\right\}$, where $L^{\infty}(G)$ is the Banach space composed of essentially-bounded measurable functions and $L(a) x(b)=x(a b)$. As is readily seen, $\left\{L^{\infty}(G), L(a)\right\}$ is not completely decomposable; more precisely, $L^{\infty}(G)$ has $C(G)$ (=the family of continuous functions) as the greatest invariant subspace in which the induced representation is completely decomposable. On the other hand, by Theorem $10\left\{L^{\infty}(G), L(a)\right\}$ has sufficiently many topological composition series. Hence, combined this fact with Theorem 5, we see that the complete decomposability practically attributes a stronger property to a uniformly bounded representation than the existence of a topological composition series, even if the representation space is a Banach space.

Finally we consider Theorem 3 . The converse of Theorem 3 is valid in the following form :

Proposition 10. Let $\{L, T(a)\}$ be a uniformly bounded representation. If $\{L$, $T(a)\}$ is completely decomposable, then $\{L, T(a)\}$ is a totally bounded representation.

Proof. Let $x$ be any element of $L$, and $U$ any neighborhood of 0 . By Lemma 1 , there exists a finite-dimensional representation $\left\{L_{\alpha}, T(a)\right\}, L_{\alpha} \subset L$, such that, for 
a suitable element $y$ belonging to $L_{\alpha}$, we have

$$
T(a) x-T(a) y \in U, \quad a \in G .
$$

Since $\left\{L_{\alpha}, T(a)\right\}$ is totally bounded, it follows immediately that $\{L, T(a)\}$ is totally bounded.

It is a remarkable fact that our discussions on complete decomposablility have been throughout restricted to uniformly bounded representations.

\section{University of Tokyo}

\section{Bibliography.}

[1] R. Arens: Duality in linear spaces, Duke Math. J. 14 (1947), 787-784.

[2] S. Bochner and J.v. Neumann: Almost periodic functions in a group II, Trans. Am. Math. Soc. 37 (1935), 21-50.

[3] N. Bourbaki: Espaces vectorieles topologiques, chap. i, ii, Act. Ind., Paris (1953).

[4] J. Dieudonné and L. Schwartz: La dualité dans les espaces (F) et (LF), Ann. Inst. Fourier Grenoble 1 (1949), 61-101.

[5] J. Dieudonné: Recent developments in the theory of locally convex topological vector space, Bull. Am. Math. Soc. 59 (1953), 495-512.

[6] W.F. Eberlein: Abstract ergodic theorems and weak almost periodic functions, Trans. Am. Math. Soc. 67 (1949), 217-240.

[7] L.H. Loomis: An introduction to abstract harmonic analysis, New York (1953).

[8] W. Maak: Abstrakte fastperiodishe Funktionen, Abh. Math. Sem. Hamb. 11 (1936), 367-380.

[9] W. Maak: Fastperiodishe Funktionen, Berlin (1951).

[10] G.W. Mackey: On convex topological spaces, Trans. Am. Math. Soc. 60 (1946), 520-537.

[11] J.v. Neumann: Almost periodic functions in a group I, Trans. Am. Math. Soc. 36 (1934), 445-492.

[12] J. v. Neumann: On complete topological spaces, Trans. Am. Math. Soc. 37 (1935), 1-20.

[13] K. Shiga: Representations of a compact group on a Banach space, Jour. Math. Soc. Japan, 7 (1955), 224-248.

[14] A. Weil: L'intégration dans les groupes topologiques et ses applications, Act. Sci. Ind., Paris $(1940)$. 